\title{
Resolvent Estrada Index of Cycles and Paths
}

\section{Bo Deng, Shouzhong Wang, Ivan Gutman}

\begin{abstract}
Let $G$ be a simple graph of order $n$. The resolvent Estrada index of $G$ is defined as $E E_{r}=\sum_{i=1}^{n}\left(1-\frac{\lambda_{i}}{n-1}\right)^{-1}$, where $\lambda_{1}, \lambda_{2}, \ldots, \lambda_{n}$ are the eigenvalues of $G$. Formulas for computing $E E_{r}$ of the cycle $C_{n}$ and the path $P_{n}$ are derived. The precision of these approximations are shown to be excellent. We also examine the difference and relations between the Estrada index and the resolvent Estrada index of $C_{n}$ and $P_{n}$.
\end{abstract}

Keywords: Resolvent Estrada index, Estrada index, spectrum (of graph), cycle, path.

\section{Introduction}

Let $G$ be a simple graph of order $n$ with vertex set $\left\{v_{1}, v_{2}, \ldots, v_{n}\right\}$. The adjacency matrix of $G$, is the square matrix $\mathbf{A}=\left(a_{i j}\right)$ of order $n$, in which $a_{i j}=1$ if the vertices $v_{i}$ and $v_{j}$ are adjacent, and $a_{i j}=0$ otherwise. The characteristic polynomial $\phi(G, \lambda)$ of the graph $G$ is the polynomial of degree $n$, defined as $\operatorname{det}\left(\lambda \mathbf{I}_{n}-\mathbf{A}\right)$, where $\mathbf{I}_{n}$ is the unit matrix of order $n$. Since $\mathbf{A}$ is real and symmetric, all its eigenvalues $\lambda_{1}, \lambda_{2}, \ldots, \lambda_{n}$ are real. These form the spectrum of the graph $G$.

For an integer $k \geq 0$, the $k$-th spectral moment of $G$ is defined as

$$
M_{k}=M_{k}(G)=\sum_{i=1}^{n} \lambda_{i}^{k} .
$$

In this paper, we are concerned with two simple and frequently encountered graphs the cycle $C_{n}$ and the path $P_{n}$. Recall that $C_{n}$ is the connected graph of order $n$ in which all vertices have degree 2. The path $P_{n}$ is the tree (= connected acyclic graph) of order $n$ in

Manuscript received October 21, 2015; accepted January 14, 2016.

Bo Deng is with Guangdong University of Petrochemical Technology, Mao Ming, Guangdong 525000, China, and Center for Combinatorics and LPMC-TJKLC, Nankai University, Tianjin 350002, China; Shouzhong Wang is with the College of Science, Guangdong University of Petrochemical Technology, Maoming, Guangdong, 525000, P. R. China; I. Gutman is with the Faculty of Science, University of Kragujevac, Serbia, and the State University of Novi Pazar, Serbia. 
which exactly two vertices have degree 1 (whereas all other vertices have degree 2 ). The spectra of $C_{n}$ and $P_{n}$ are well known [4] (see below).

In 2000, Ernesto Estrada introduced a structural invariant based on the spectral moments, defined as [6]

$$
E E=E E(G)=\sum_{k=0}^{\infty} \frac{M_{k}}{k !}
$$

which eventually was named "Estrada index" [14]. Applications of the Estrada index range from the description of folding of protein molecules $[6,7]$ to measuring the centrality of complex (communication, social, metabolic, etc.) networks $[9,8]$. This graph-spectrumbased invariant was also subject of extensive mathematical studies, for review see [10].

Recently, Estrada and Higham [8] modified the quantity occurring on the right-hand side of Eq. (1), and considered the spectrum-based invariant

$$
E E_{r}=E E_{r}(G)=\sum_{k=0}^{\infty} \frac{M_{k}}{(n-1)^{k}} .
$$

Bearing in mind that $\left|\lambda_{i}\right|<n-1$ holds for for all eigenvalues of all graphs of order $n$, except in the case of the $n$-vertex complete graph [4], the right-hand side summation in Eq. (2) is convergent. Thus, $E E_{r}$ is well defined for all graphs, except for the complete graphs. It is easy to verify that

$$
E E_{r}=\sum_{i=1}^{n} \frac{n-1}{n-1-\lambda_{i}}=\sum_{i=1}^{n}\left(1-\frac{\lambda_{i}}{n-1}\right)^{-1}
$$

The expression on the right-hand side of Eq. (3) indicates that $E E_{r}$ is a resolvent-operatorbased quantity [1], in view of which $E E_{r}$ has been named resolvent Estrada index.

Although $E E_{r}$ has many properties analogous to those of $E E$, the two indices are distinct in essence $[8,3]$. In what follows, we will show additional evidence to support this view.

At the present moment, there are only a few mathematical and computational studies of the resolvent Estrada index [3, 2, 11, 12]. Some of the basic properties of $E E_{r}$ have been established, but numerous open problems (many stated in form of conjectures [12]) await to be solved in the future. The present work is aimed at contributing towards partially filling this gap.

Cycles and paths play an important role in researching the resolvent Estrada index. In particular, we have:

Theorem 1 [11] Among all connected graphs of order $n(n \geq 1)$, the path $P_{n}$ has minimal resolvent Estrada index.

In order to find the graph with second-minimal $E E_{r}$-value, the tree $P_{n-1}(j)$ had to be considered [5,11], where $P(n-1)(j)$ is obtained by attaching a pendent vertex at position $j$ of the path $P_{n-1}$. Then it follows: 
Theorem 2 [11] Among all connected graphs of order $n(n \geq 4)$, the tree $P_{n-1}(2)$ has the second-minimal resolvent Estrada index.

For a complete proof of Theorem 2, it was necessary to show that [11]

$$
E E_{r}\left(P_{n-1}(2)\right)<E E_{r}\left(C_{n}\right)
$$

The latter inequality holds because $C_{n}$ possesses many more self-returning walks than $P_{n-1}(2)$ and some of the odd spectral moments of $C_{n}$ are greater than zero if $n$ is odd. Similarly, when $j \geq 3$, the condition $E E_{r}\left(P_{n-1}(j)\right)<E E_{r}\left(C_{n}\right)$ is necessary for establishing the $j$-th minimal resolvent Estrada index among all connected graphs of order $n$ [11].

In [12], based on extensive computer work, it has been shown that the cycle $C_{n}$ has smallest $E E_{r}$-value among all connected unicyclic graphs of order $n(n \geq 3)$.

Motivated by the above results, we now offer a simple method for computing the resolvent Estrada indices of $P_{n}$ and $C_{n}$. Before presenting it, we outline the analogous formulas for the ordinary Estrada index.

\section{Estrada indices of cycles and paths}

Graovac and one of the present authors [13] showed that the Estrada indices of the cycles and paths can be approximated as

$$
E E\left(C_{n}\right) \approx n I_{0}
$$

and

$$
E E\left(P_{n}\right) \approx(n+1) I_{0}-\cosh (2)
$$

where $I_{0}=\sum_{k \geq 0} 1 /(k !)^{2}=2.27958530 \ldots$ Hence, in the case of cycles and paths, $E E$ can be calculated easily.

In Fig. 1 are presented the $E E\left(C_{n}\right)$ - and $E E\left(P_{n}\right)$-values for $1 \leq n<16$. As it can be seen, except for the first few values of $n$, the plots of $E E\left(C_{n}\right)$ and $E E\left(P_{n}\right)$ are practically parallel, and $E E\left(C_{n}\right)$ is always greater than $E E\left(P_{n}\right)$. This is in full agreement with Eqs. (4) and (5). 


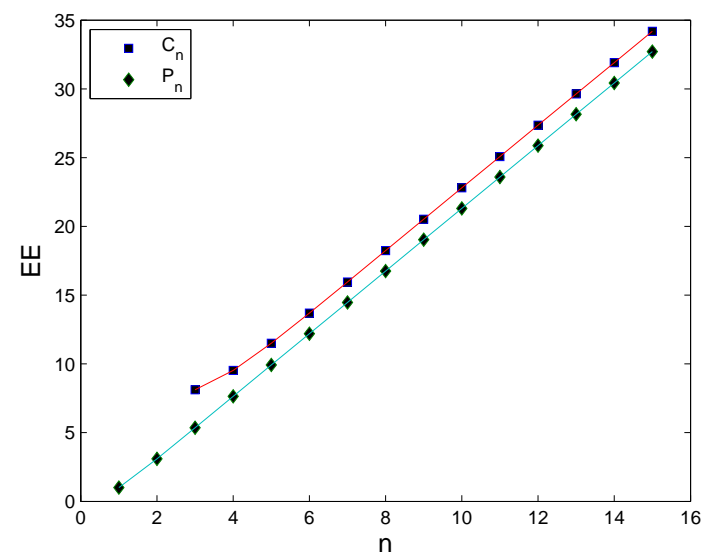

Fig. 1. $E E\left(C_{n}\right)$ (upper) and $E E\left(P_{n}\right)$ (lower), plotted versus $n$.

\section{Resolvent Estrada index of cycles}

It is well known [4] that the spectrum of the cycle $C_{n}$ consists of the numbers $2 \cos (2 i \pi / n), i=$ $1,2, \ldots, n$. In view of this,

$$
\begin{aligned}
E E_{r}\left(C_{n}\right) & =\sum_{i=1}^{n}\left(1-\frac{2 \cos \frac{2 i \pi}{n}}{n-1}\right)^{-1} \\
& =\sum_{i=1}^{n}\left[1+\frac{2 \cos \frac{2 i \pi}{n}}{n-1}+\left(\frac{2 \cos \frac{2 i \pi}{n}}{n-1}\right)^{2}+\left(\frac{2 \cos \frac{2 i \pi}{n}}{n-1}\right)^{3}\right. \\
& \left.+\left(\frac{2 \cos \frac{2 i \pi}{n}}{n-1}\right)^{4}+\left(\frac{2 \cos \frac{2 i \pi}{n}}{n-1}\right)^{5}+\left(\frac{2 \cos \frac{2 i \pi}{n}}{n-1}\right)^{6}+\cdots\right]
\end{aligned}
$$

One can see that the angles $2 i \pi / n$ uniformly cover the interval $[0,2 \pi]$ when $i=1,2, \ldots, n$. 
Thus, the following integral approximation is applicable:

$$
\begin{aligned}
E E_{r}\left(C_{n}\right) & =n+\sum_{i=1}^{n} \frac{2 \cos \frac{2 i \pi}{n}}{n-1}+\sum_{i=1}^{n}\left(\frac{2 \cos \frac{2 i \pi}{n}}{n-1}\right)^{2}+\sum_{i=1}^{n}\left(\frac{2 \cos \frac{2 i \pi}{n}}{n-1}\right)^{3} \\
& +\sum_{i=1}^{n}\left(\frac{2 \cos \frac{2 i \pi}{n}}{n-1}\right)^{4}+\sum_{i=1}^{n}\left(\frac{2 \cos \frac{2 i \pi}{n}}{n-1}\right)^{5}+\sum_{i=1}^{n}\left(\frac{2 \cos \frac{2 i \pi}{n}}{n-1}\right)^{6}+\cdots \\
& \approx n+\frac{n}{2 \pi(n-1)} \int_{0}^{2 \pi} 2 \cos x d x+\frac{n}{2 \pi(n-1)^{2}} \int_{0}^{2 \pi}(2 \cos x)^{2} d x \\
& +\frac{n}{2 \pi(n-1)^{3}} \int_{0}^{2 \pi}(2 \cos x)^{3} d x+\frac{n}{2 \pi(n-1)^{4}} \int_{0}^{2 \pi}(2 \cos x)^{4} d x \\
& +\frac{n}{2 \pi(n-1)^{5}} \int_{0}^{2 \pi}(2 \cos x)^{5} d x+\frac{n}{2 \pi(n-1)^{6}} \int_{0}^{2 \pi}(2 \cos x)^{6} d x
\end{aligned}
$$

By direct computation, we obtain

$$
E E_{r}\left(C_{n}\right) \approx n+\frac{2 n}{(n-1)^{2}}+\frac{6 n}{(n-1)^{4}}+\frac{20 n}{(n-1)^{6}} .
$$

The precision of the approximate expression (6) can be seen from the data in Table 1. Except for the first few values of $n$, the resolvent Estrada indices of cycles are excellently reproduced by Eq. (6). Thus, its accuracy is on two, four, and six decimal places for, respectively, $n \geq 5, n \geq 7$, and $n \geq 11$. This is more than sufficient for any standard application of $E E_{r}\left(C_{n}\right)$.

\section{Resolvent Estrada index of paths}

In a similar way as in the case of $C_{n}$, yet somewhat more complicated, we get an approximate expression for $E E_{r}\left(P_{n}\right)$. The spectrum of $P_{n}$ consists of the numbers $2 \cos [i \pi /(n+$ 


\begin{tabular}{||l|l|l|l|l||}
\hline$n$ & $E E_{r}\left(C_{n}\right)$ & $E E_{r}\left(C_{n}\right)_{\text {approx }}$ & $E E_{r}\left(P_{n}\right)$ & $E E_{r}\left(P_{n}\right)_{\text {approx }}$ \\
\hline 3 & & & 5.0000025 & 4.7500000 \\
5 & 5.6000000 & 5.2949246 & 4.9090909 & 4.8888889 \\
6 & 6.54764737 & 5.7666016 & 5.5948719 & 5.5917969 \\
7 & 7.4246863 & 6.5452800 & 6.4471502 & 6.4464640 \\
8 & 8.3479907 & 8.3478823 & 8.3025966 & 8.3025270 \\
9 & 9.2951602 & 9.2951202 & 9.2612890 & 9.2612610 \\
10 & 10.2564519 & 10.2564349 & 10.2301490 & 10.2301366 \\
11 & 11.2268280 & 11.2268200 & 11.2057821 & 11.2057760 \\
12 & 12.2034043 & 12.2034003 & 12.1861666 & 12.1861635 \\
13 & 13.1844062 & 13.1844042 & 13.1700201 & 13.1700183 \\
14 & 14.1686808 & 14.1686780 & 14.1564870 & 14.1564860 \\
15 & 15.1554446 & 15.1554438 & 15.1449742 & 15.1449736 \\
16 & 16.1441471 & 16.1441466 & 16.1350567 & 16.1350563 \\
17 & 17.1343895 & 17.1343892 & 17.1264217 & 17.1264215 \\
18 & 18.1258756 & 18.1258755 & 18.1188337 & 18.1188335 \\
19 & 19.1183810 & 19.1183811 & 19.1121118 & 19.1121117 \\
20 & 20.1117327 & 20.1117326 & 20.1061149 & 20.1061148 \\
21 & 21.1057942 & 21.1057941 & 21.1007309 & 21.1007309 \\
22 & 22.1004571 & 22.1004571 & 22.0958701 & 22.0958700 \\
23 & 23.0956344 & 23.0956345 & 23.0914592 & 23.0914592 \\
24 & 24.0912551 & 24.0912551 & 24.0874383 & 24.0874383 \\
25 & 25.0872602 & 25.0872603 & 25.0837577 & 25.0837577 \\
26 & 26.0836015 & 26.0836015 & 26.0803757 & 26.0803757 \\
27 & 27.0802379 & 27.0802379 & 27.0772573 & 27.0772573 \\
28 & 28.0771351 & 28.0771351 & 28.0743727 & 28.0743727 \\
29 & 29.0742640 & 29.0742639 & 29.0716965 & 29.0716965 \\
30 & 30.0715991 & 30.0715991 & 30.0692068 & 30.0692068 \\
\hline
\end{tabular}

Table 1. Exact and approximate values of resolvent Estrada indices of $C_{n}$ and $P_{n}$.

1)], $i=1,2, \ldots, n[4]$. In view of this,

$$
\begin{aligned}
E E_{r}\left(P_{n}\right) & =\sum_{i=1}^{n}\left(1-\frac{2 \cos \frac{i \pi}{n+1}}{n-1}\right)^{-1} \\
& =\sum_{i=1}^{n}\left[1+\left(\frac{2 \cos \frac{i \pi}{n+1}}{n-1}\right)+\left(\frac{2 \cos \frac{i \pi}{n+1}}{n-1}\right)^{2}+\left(\frac{2 \cos \frac{i \pi}{n+1}}{n-1}\right)^{3}\right. \\
& \left.+\left(\frac{2 \cos \frac{i \pi}{n+1}}{n-1}\right)^{4}+\left(\frac{2 \cos \frac{i \pi}{n+1}}{n-1}\right)^{5}+\left(\frac{2 \cos \frac{i \pi}{n+1}}{n-1}\right)^{6}+\cdots\right] .
\end{aligned}
$$


Since the angles $i \pi /(n+1)$ do not cover the entire interval $[0, \pi]$, the missing near-zero and near- $\pi$ contributions need to be compensated when applying an integral approximation. Bearing this in mind, we can proceed as follows. Let $m>0$ be an integer. Then,

$$
\begin{aligned}
\sum_{i=1}^{n}\left(\frac{2 \cos \frac{i \pi}{n+1}}{n-1}\right)^{m} & =\frac{1}{2} \sum_{i=0}^{n}\left(\frac{2 \cos \frac{i \pi}{n+1}}{n-1}\right)^{m}+\frac{1}{2} \sum_{i=1}^{n+1}\left(\frac{2 \cos \frac{i \pi}{n+1}}{n-1}\right)^{m} \\
& -\frac{1}{2}\left[\left(\frac{2}{n-1}\right)^{m}+\left(\frac{-2}{n-1}\right)^{m}\right] \\
& \approx \frac{n+1}{2 \pi(n-1)^{m}} \int_{0}^{\pi}(2 \cos x)^{m} d x+\frac{n+1}{2 \pi(n-1)^{m}} \int_{0}^{\pi}(2 \cos x)^{m} d x \\
& -\frac{1}{2}\left[\left(\frac{2}{n-1}\right)^{m}+\left(\frac{-2}{n-1}\right)^{m}\right] \\
& =\frac{n+1}{\pi(n-1)^{m}} \int_{0}^{\pi}(2 \cos x)^{m} d x-\frac{1}{2}\left[\left(\frac{2}{n-1}\right)^{m}+\left(\frac{-2}{n-1}\right)^{m}\right]
\end{aligned}
$$

Note that $\sum_{i=1}^{n}\left(\frac{2 \cos \frac{i \pi}{n+1}}{n-1}\right)^{m}$ is approximately equal to 0 when $m$ is odd. Therefore,

$$
\begin{aligned}
E E_{r}\left(P_{n}\right) & \approx n+\frac{n+1}{\pi(n-1)^{2}} \int_{0}^{\pi}(2 \cos x)^{2} d x-\frac{2^{2}}{(n-1)^{2}}+\frac{n+1}{\pi(n-1)^{4}} \int_{0}^{\pi}(2 \cos x)^{4} d x \\
& -\frac{2^{4}}{(n-1)^{4}}+\frac{n+1}{\pi(n-1)^{6}} \int_{0}^{\pi}(2 \cos x)^{6} d x-\frac{2^{6}}{(n-1)^{6}}
\end{aligned}
$$

This finally yields

$$
E E_{r}\left(P_{n}\right) \approx n+\frac{2 n-2}{(n-1)^{2}}+\frac{6 n-10}{(n-1)^{4}}+\frac{20 n-44}{(n-1)^{6}} .
$$

In Table 1 are also given the exact and approximate values of $E E_{r}\left(P_{n}\right)$. We see that the precision of the approximation (7) is remarkably good. Comparing the values of $E E_{r}\left(C_{n}\right)$ and $E E_{r}\left(P_{n}\right)$, it becomes evident that, except for the first few values of $n$, these two resolvent Estrada indices are almost equal. The very same conclusion is obtained by comparing the expressions (6) and (7). 


\section{Comparing Estrada and resolvent Estrada indices}

In this section, we examine the relations and difference between the Estrada index and the resolvent Estrada index in the case of $C_{n}$ and $P_{n}$.

As seen from Eqs. (1) and (2), the resolvent Estrada index depends on all eigenvalues or all spectral moments of the underlying graph. Yet, as shown by our formulas (6) and (7), in the case of cycles and paths this index can be calculated (approximately, but with very high precision) from just the number of vertices, $n$.

In Fig. 2 are shown $E E_{r}\left(C_{n}\right)$ and $E E_{r}\left(P_{n}\right)$ for $2<n<16$. We see that, except for the first few values of $n, E E_{r}\left(C_{n}\right)$ and $E E_{r}\left(P_{n}\right)$ are almost equal. This finding is in full agreement with the approximate expressions deduced in Sections 3 and 4.

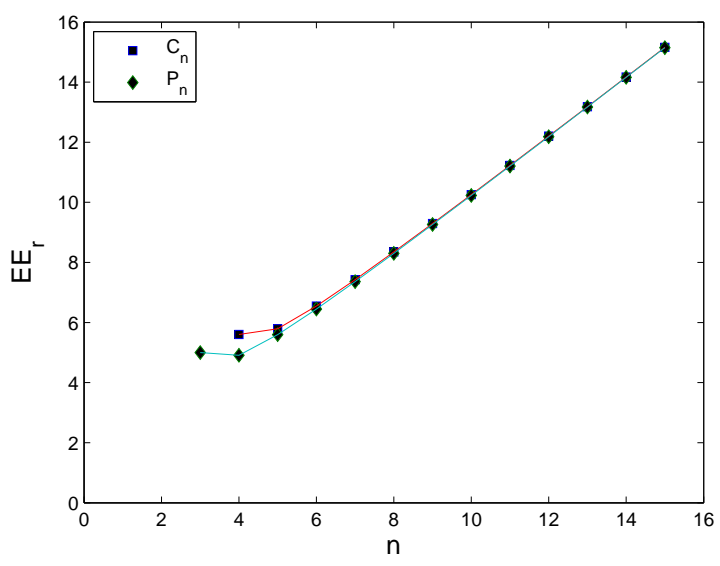

Fig. 2. $E E_{r}\left(C_{n}\right)$ and $E E_{r}\left(P_{n}\right)$, plotted versus $n$.

By comparing Figs. 1 and 2, a remarkable difference is envisaged between the Estrada index and the resolvent Estrada index in the case of cycles and paths. The reason for this difference can be explained as follows.

From the expressions (4) and (5), it is immediately seen that, except for the first few values of $n, E E\left(C_{n}\right)$ and $E E\left(P_{n}\right)$ are linear functions of $n$. Due to the same slope $I_{0}$ of Eqs. (4) and (5), the two lines shown in Fig. 1 are parallel. The difference between the two lines is given by the term $\cosh (2)-I_{0}$, which is a fixed constant, and is approximately equal to 1.4826 .

On the other hand, the expressions (6) and (7) for the resolvent Estrada indices of $C_{n}$ and $P_{n}$ are non-linear functions of $n$, although their deviation from linearity rapidly vanishes 
with increasing $n$. The difference between (6) and (7) is

$$
\frac{2}{(n-1)^{2}}+\frac{10}{(n-1)^{4}}+\frac{44}{(n-1)^{6}}
$$

which rapidly tends to zero for $n \rightarrow \infty$. This implies that the two lines presented in Fig. 2 gradually tend to become linear and tend to coincide as $n$ increases. That this happens very fast is seen from Fig. 2 and the data given in Table 1.

By comparing expressions (4) and (6) and bearing in mind that $I_{0} \approx 2.27958530$, we easily see that

$$
E E_{r}\left(C_{n}\right)<E E\left(C_{n}\right)
$$

holds provided $n \geq 4$.

Indeed, if $n \geq 4$, then

$$
\frac{2 n}{(n-1)^{2}}+\frac{6 n}{(n-1)^{4}}+\frac{20 n}{(n-1)^{6}} \leq 3 .
$$

and then by (4) and (6),

$$
n+\frac{2 n}{(n-1)^{2}}+\frac{6 n}{(n-1)^{4}}+\frac{20 n}{(n-1)^{6}} \leq n+3 \leq 2 n<n I_{0}
$$

implying the inequality (8).

In an analogous manner, for $n \geq 3$, we get

$$
E E_{r}\left(P_{n}\right)<E E\left(P_{n}\right) .
$$

Inequalities (8) and (9) shed some light on the relations between the Estrada and the resolvent Estrada indices. It remains a challenge for the future to investigate whether the inequality $E E_{r}(G)<E E(G)$ holds for other graphs $G$, or - perhaps - for all graphs. If not, then it would be interesting to find graphs for which $E E_{r}(G)=E E(G)$.

Acknowledgements. This research was supported by the Science and Technology Project of Maoming City no. 001-423217050-5020, and by the Introduction of Talent Project no. 513085 .

\section{References}

[1] M. Benzi, P. Boito, Quadrature rule-based bounds for functions of adjacency matrices, Lin. Algebra Appl., Vol. 433, (2010), 637-652. 
[2] X. Chen, J. Qian, Bounding the resolvent Estrada index of a graph, J. Math. Study, Vol. 45, 2 (2012), 159-166.

[3] X. Chen, J. Qian, On resolvent Estrada index, MATCH Commun. Math. Comput. Chem., Vol. 73, 1 (2015), 163-174.

[4] D. Cvetković, M. Doob, H. Sachs, Spectra of Graphs - Theory and Application, Academic Press, New York, 1980.

[5] H. Deng, A note on the Estrada index of trees, MATCH Commun. Math. Comput. Chem., Vol. 62, 3 (2009), 607-610.

[6] E. Estrada, Characterization of 3D molecular structure, Chem. Phys. Lett., Vol. 319, (2000), 713-718.

[7] E. Estrada, Characterization of the folding degree of proteins, Bioinformatics, Vol. 18, (2002), 697-704.

[8] E. Estrada, D. J. Higham, Network properties revealed through matrix functions, SIAM Rev., Vol. 52 (2010) 696-714.

[9] E. Estrada, J. A. Rodríguez-Velázquez, Subgraph centrality in complex networks, Phys. Rev. E, Vol. 71, (2005), 056103.

[10] I. Gutman, H. Deng, S. Radenković, The Estrada index: An updated survey, in: D. Cvetković, I. Gutman (Eds.), Selected Topics on Applications of Graph Spectra, Math. Inst., Beograd, 2011, pp. 155-174.

[11] I. Gutman B. Furtula, X. Chen, J. Qian, Graphs with smallest resolvent Estrada indices, MATCH Commun. Math. Comput. Chem., Vol. 73, 1 (2015), 267-270.

[12] I. Gutman, B. Furtula, X. Chen, J. Qian, Resolvent Estrada index - computational and mathematical studies, MATCH Commun. Math. Comput. Chem., Vol. 74, 3 (2015), 431-440.

[13] I. Gutman, A. Graovac, Estrada indices of cycles and paths, Chem. Phys. Lett., Vol. 463, (2007), 294-296.

[14] J. A. de la Peña, I. Gutman, J. Rada, Estimating the Estrada index, Lin. Algebra Appl., Vol. 427, (2007), 70-76. 